\title{
Pattern of HIV testing and multiple sexual partnerships among men who have sex with men in China
}

Eric P F Chow ${ }^{1,2,3,4}$, Jun Jing ${ }^{2}$, Yuji Feng ${ }^{5}$, Dai Min ${ }^{5}$, Jun Zhang ${ }^{2}$, David P Wilson' ${ }^{1}$, Xiaohu Zhang ${ }^{2,6^{*}}$ and Lei Zhang ${ }^{1,2^{*}}$

\begin{abstract}
Background: Men who have sex with men (MSM) are a hidden but emerging population susceptible to HIV infection against a background of rapidly increasing HIV prevalence in China. Low HIV testing levels and multiple partnerships among MSM are two major contributing factors to HIV transmission.

Methods: We conducted a cross-sectional survey among 447 Chinese MSM in Changsha and Tianjin cities from November to December 2011 using an anonymous questionnaire. We aim to investigate (1) the trend of HIV testing rates among Chinese MSM during 2009 to 2011; and (2) the patterns of multiple sexual relationships with male, female and commercial partners.

Results: The self-reported past-12-months HIV testing level among Chinese MSM increased from 16.6\% in 2009 to $46.3 \%$ in 2010 and $58.6 \%$ in $2011\left(x^{2}=173.49, p<0.001\right)$. Compared with men who have tested for HIV, the never-tested MSM were generally younger, never married, students, and more likely to have unprotected anal intercourse with non-commercial male partners. Furthermore, 21.3\% (56/263) MSM reported having multiple regular male and female sexual partnerships and 6.2\% (16/257) reported having commercial male partners in the past six months. However, individuals who were never-tested for HIV are consistently less likely to engage in multiple sexual relationships.
\end{abstract}

Conclusions: HIV testing rates have increased substantially among Chinese MSM in the period 2009-2011, although significant barriers to testing remain. Multiple sexual partnerships, and especially bisexual behaviours, are common among Chinese MSM.

Keywords: Men who have sex with men (MSM), China, HIV testing, Sexual behaviours

\section{Background}

The predominant mode of HIV transmission in China has shifted in the last decade from sharing of injecting equipment among drug users to sexual transmission [1]. The epidemic has emerged and spread rapidly among men who have sex with men (MSM) in recent years [1-3]. The national prevalence of HIV among Chinese MSM has quadrupled from $1.4 \%$ in 2001 to $6.3 \%$ in 2011 [4,5] and the proportion of annual HIV incidence attributable to

\footnotetext{
* Correspondence: zzhenhan@gmail.com; lzhang@kirby.unsw.edu.au ${ }^{2}$ Comprehensive AIDS Research Center, Tsinghua University, Beijing, China ${ }^{1}$ The Kirby Institute, University of New South Wales, Sydney, New South Wales, Australia

Full list of author information is available at the end of the article
}

homosexual contacts increased from $12.2 \%$ in 2007 to $32.5 \%$ in 2009 [2]. Chinese MSM are a complex population, as the majority of MSM not only participate in homosexual activities [6] but also in frequent heterosexual activities and the commercial sex trade [6-8]. Multiple sexual partnerships with both men and women are common among Chinese MSM and approximately $70-90 \%$ will eventually enter heterosexual marriage [9-11]. Reported low condom use between MSM [12] and regular female partners [7] suggests a potential bridge of HIV infection to the general female population through their multiple sexual partnerships [13,14].

HIV testing is a primary and effective strategy to identify infections; early diagnosis of HIV also enables timely

\section{Ciomed Central}


treatments in infected individuals [15]. Despite the recent report of substantial improvements, the national level of annual HIV testing rate among Chinese MSM (50.4\% in 2011 [5]) remains substantially lower than other developed countries such as Norway (56\%) and Australia (60$70 \%)[16,17]$. In addition, mathematical modelling of HIV transmission among Chinese MSM suggests that $87 \%$ of HIV-positive MSM are not aware of their infection [18], indicating a very high undiagnosed rate compared with MSM in developed countries [15,19-21]. Understanding the underlying socio-demographic and behavioural contributing factors to HIV testing among MSM is crucial for effective prevention efforts.

It has become apparent that the patterns of HIV testing and multiple types of partnerships are two key but underinvestigated areas among MSM in China. In the light of this, we conducted a retrospective cross-sectional study to investigate (i) the trend of annual and repeated HIV testing among MSM during 2009-2011; and (ii) their patterns of sexual behaviours with multiple types of sexual partners (non-commercial male, female and commercial male) in the past six months.

\section{Methods}

\section{Study subjects and recruitment}

A community-based cross-sectional study among MSM was conducted in Changsha and Tianjin cities from November to December 2011. Potential participants were recruited through venue-based convenience sampling and peer-referral. Peer recruiters were the volunteers from local MSM community-based organizations (i.e. 'Tianjin Deep Blue Voluntary working group' in Tianjin; 'Changsha Zonda-Sunlight Working Party' and 'Zuo An Cai Hong' in Changsha). Ten volunteers were trained for outreach to recruit participants in well-known MSM hotspots including gay bars, clubs, saunas and bathhouses. Each volunteer spent three to four hours on outreach recruitment on every weekend evening throughout the 2-month period. In addition, peer recruiters and participants were also asked to refer their peers to participate in this study. Participants were eligible if they were (i) aged 15 or over and had engaged in anal or oral sex with men in the past 12 months; and (ii) able to recall whether they had ever been tested for HIV in their lifetime. Participants were asked to complete an anonymous questionnaire of approximately 15-20 minutes duration through face-to-face interviews. All participants, if necessary, were invited or referred to the voluntary counselling and testing (VCT) service that offered by the local community-based organizations.

\section{Measures}

The questionnaire covered aspects of demographic characteristics, HIV testing history and sexual behaviours (see Additional file 1). Study participants were asked about demographic characteristics including age, marital status (never married, married or cohabiting with female, divorced or widowed, cohabiting with male, and other), residency (local, and non-local), educational level (junior high school or below, senior high school, and college or above), occupation (student, self-employed, employed in stateowned or foreign companies, and other) and self-reported sexual orientation (homosexual, heterosexual, bisexual and unsure).

Questions related to HIV testing history and behaviours were stratified according to whether the participants had ever received an HIV test. In the case of negative responses, the participants were asked about the reason(s) for not having an HIV test in the past. A list of fourteen possible reasons was provided and participants could give more than one answer. Participants who indicated that they had ever had an HIV test were asked to recall the context and frequency of HIV testing in the previous three years (2009-2011). All participants were asked about their activity with various types of sexual partnerships (noncommercial male, commercial male and non-commercial female), including specific questions such as (i) whether they had had sex with different types of sexual partners in the past six months; (ii) condom use during the last anal/ vaginal intercourse; (iii) awareness of HIV serostatus of the last sexual partner; and (iv) how long ago had the last three episodes of sexual intercourse occurred.

\section{Statistical analysis}

Questionnaire data were double entered and checked in Microsoft Access 2007 (Microsoft Corporation, Washington, USA). Participants were categorised into three independent groups (tested within the past 12 months; tested more than 12 months ago; and never tested) [22] to investigate the differences between the three groups, in demographic characteristics and sexual risk behaviours. Descriptive statistics and frequency distribution of the sample were calculated. We employed Chi-Square test to investigate the association between the dependent variable and the categorical study variables; continuous study variables were tested by using one-way analysis of variance (ANOVA). Significance level of 0.05 was used for all statistical tests. Gap durations (i.e. number of days) between the last two consecutive episodes of sexual intercourse in different types of partnerships were also estimated. All statistical analyses were performed in STATA version 12.0 (StataCrop, Texas, USA).

\section{Ethical considerations}

Verbal and written consent procedures were given to the study participants and they had the right to discontinue the survey at any time. This study was approved by the Human Research Ethics Committee of the University of 
New South Wales (HC12450) and the Institutional Review Board of the Tsinghua University (\#00201204).

\section{Results}

\section{Demographic characteristics}

A total of 455 MSM were recruited in this study. Eight were excluded as they could not recall whether they had been tested for HIV. The remaining 447 participants met the eligibility criteria and completed the questionnaire; of these, 238 were from Changsha city and 209 from Tianjin city. Although significant differences in demographic characteristics were observed between the two groups (Table 1), HIV testing behaviours did not significantly differ $\left(\chi^{2}=1.26, p=0.53\right)$. Since the withinsample correlation of demographic characteristics with the testing behaviours were similar but with different compositions in two cities independently (see Additional file 2), data from the two cities were pooled together for the analyses of associated factors of HIV testing (Table 2). Of the 447 participants, $262(58.6 \%)$ were tested within the past 12 months; 95 (21.3\%) were tested more than 12 months previously; and 90 (20.1\%) had never been tested. MSM who had never been tested were significantly younger ( $26.42 \pm 8.64$ years) than those who had

Table 1 Socio-demographic characteristic and sexual behavioural factors that associated with HIV testing among Chinese MSM

\begin{tabular}{|c|c|c|c|c|c|c|c|c|}
\hline \multirow[t]{2}{*}{ Characteristics } & \multicolumn{2}{|c|}{$\begin{array}{c}\text { Total } \\
(\mathrm{N}=447)\end{array}$} & \multicolumn{2}{|c|}{$\begin{array}{l}\text { Changsha } \\
(\mathrm{N}=238)\end{array}$} & \multicolumn{2}{|c|}{$\begin{array}{c}\text { Tianjin } \\
(\mathrm{N}=209)\end{array}$} & \multirow[t]{2}{*}{$x^{2}$} & \multirow[t]{2}{*}{$P$-value } \\
\hline & $n$ & $\%$ & $n$ & $\%$ & $n$ & $\%$ & & \\
\hline Age & & & & & & & 6.80 & $0.03^{*}$ \\
\hline$<20$ & 30 & $6.7 \%$ & 22 & $9.2 \%$ & 8 & $3.8 \%$ & & \\
\hline 20-39 & 361 & $80.8 \%$ & 182 & $76.5 \%$ & 179 & $85.6 \%$ & & \\
\hline$\geq 40$ & 54 & $12.1 \%$ & 32 & $13.4 \%$ & 22 & $10.5 \%$ & & \\
\hline Missing & 2 & $0.4 \%$ & 2 & $0.8 \%$ & 0 & $0 \%$ & & \\
\hline Marital status & & & & & & & 3.02 & 0.08 \\
\hline Never married & 287 & $64.2 \%$ & 142 & $59.7 \%$ & 145 & $69.4 \%$ & & \\
\hline Married/Cohabiting/divorced/widowed & 153 & $34.2 \%$ & 89 & $37.4 \%$ & 64 & $30.6 \%$ & & \\
\hline Others/Missing & 7 & $1.6 \%$ & 7 & $2.9 \%$ & 0 & $0 \%$ & & \\
\hline Residency & & & & & & & 20.97 & $<0.001^{* * *}$ \\
\hline Local & 216 & $48.3 \%$ & 89 & $37.4 \%$ & 127 & $60.8 \%$ & & \\
\hline Non-local & 222 & $49.7 \%$ & 140 & $58.8 \%$ & 82 & $39.2 \%$ & & \\
\hline Missing & 9 & $2.0 \%$ & 9 & $3.8 \%$ & 0 & $0 \%$ & & \\
\hline Education Level & & & & & & & 37.411 & $<0.001^{* * *}$ \\
\hline Junior high \& lower & 32 & $7.2 \%$ & 9 & $3.8 \%$ & 23 & $11.0 \%$ & & \\
\hline Senior high & 141 & $31.5 \%$ & 52 & $21.8 \%$ & 89 & $42.6 \%$ & & \\
\hline College \& above & 273 & $61.1 \%$ & 176 & $73.9 \%$ & 97 & $46.4 \%$ & & \\
\hline Missing & 1 & $0.2 \%$ & 1 & $0.4 \%$ & 0 & $0 \%$ & & \\
\hline Occupation & & & & & & & 0.02 & 0.90 \\
\hline Student/Others & 234 & $52.3 \%$ & 124 & $52.1 \%$ & 110 & $52.6 \%$ & & \\
\hline Employed & 209 & $46.8 \%$ & 112 & $47.1 \%$ & 97 & $46.4 \%$ & & \\
\hline Missing & 4 & $0.9 \%$ & 2 & $0.8 \%$ & 2 & $1.0 \%$ & & \\
\hline Self-identified sexual identity & & & & & & & 16.05 & $<0.001^{* * *}$ \\
\hline Homosexual & 353 & $79.0 \%$ & 201 & $84.5 \%$ & 152 & $72.7 \%$ & & \\
\hline Heterosexual/Bisexual & 83 & $18.6 \%$ & 27 & $11.3 \%$ & 56 & $26.8 \%$ & & \\
\hline Unsure/Missing & 11 & $2.5 \%$ & 10 & $4.2 \%$ & 1 & $0.5 \%$ & & \\
\hline HIV testing behaviour & & & & & & & 1.26 & 0.53 \\
\hline Tested within the past 12 months & 262 & $58.6 \%$ & 145 & $60.9 \%$ & 117 & $56.0 \%$ & & \\
\hline Tested more than 12 months ago & 95 & $21.3 \%$ & 49 & $20.6 \%$ & 46 & $22.0 \%$ & & \\
\hline Never tested & 90 & $20.1 \%$ & 44 & $18.5 \%$ & 46 & $22.0 \%$ & & \\
\hline
\end{tabular}

Note: ${ }^{*} p<0.05,{ }^{* *} p<0.01$ and ${ }^{* * *} p<0.001$. 
Table 2 Socio-demographic characteristic and sexual behavioural factors that associated with HIV testing among Chinese MSM Tested within the Tested more than 12 Never tested $X^{2} \quad P$-value past 12 months months ago

$\begin{array}{cccc}(\mathrm{N}=262 ; 58.6 \%) & & (\mathrm{N}=95 ; 21.3 \%) & \frac{(\mathrm{N}=90 ; 20.1 \%)}{n}\end{array}$

Demographic characteristics

Age

$13.950 .01^{* *}$

$<20$

20-39

$$
\geq 40
$$

Missing

Marital status

Never married

Married/cohabiting/divorced/widowed

Others/Missing

Residency

Local

Non-local

Missing

Recruitment location

Changsha

Tianjin

Education Level

Junior high \& lower

Senior high

College \& above

Missing

Occupation

Student/Others

Employed

Missing

Self-identified sexual identity

Homosexual

Heterosexual/Bisexual

Unsure/Missing

Homosexual behaviours (non-commercial)

Had sex with male partners in the past 6 months

\section{No}

Yes

Used condom during the last anal intercourse

$$
\text { No }
$$

Yes

$\begin{array}{cccccc}16 & 6.1 \% & 1 & 1.1 \% & 13 & 14.4 \% \\ 214 & 81.7 \% & 80 & 84.2 \% & 67 & 74.4 \% \\ 30 & 11.5 \% & 14 & 14.7 \% & 10 & 11.1 \% \\ 2 & 0.8 \% & 0 & 0.0 \% & 0 & 0.0 \%\end{array}$

$16.88<0.001^{* * *}$

$\begin{array}{cccccc}163 & 62.2 \% & 52 & 54.7 \% & 72 & 80.0 \% \\ 95 & 36.3 \% & 43 & 45.3 \% & 15 & 16.7 \% \\ 4 & 1.5 \% & 0 & 0.0 \% & 3 & 3.3 \%\end{array}$

$\begin{array}{llllll}122 & 46.6 \% & 43 & 45.3 \% & 51 & 56.7 \%\end{array}$

$\begin{array}{llllll}134 & 51.1 \% & 51 & 53.7 \% & 37 & 41.1 \%\end{array}$

$\begin{array}{llllll}6 & 2.3 \% & 1 & 1.1 \% & 2 & 2.2 \%\end{array}$

$1.26 \quad 0.53$

$\begin{array}{llllll}216 & 82.4 \% & 43 & 45.3 \% & 51 & 56.7 \%\end{array}$

$\begin{array}{llllll}222 & 84.7 \% & 51 & 53.7 \% & 37 & 41.1 \%\end{array}$

$\begin{array}{lll} & 4.94 & 0.29\end{array}$

$\begin{array}{llllll}24 & 9.2 \% & 4 & 4.2 \% & 4 & 4.4 \%\end{array}$

$\begin{array}{llllll}76 & 29.0 \% & 33 & 34.7 \% & 32 & 35.6 \%\end{array}$

$\begin{array}{llllll}162 & 61.8 \% & 58 & 61.1 \% & 53 & 58.9 \%\end{array}$

$\begin{array}{llllll}0 & 0.0 \% & 0 & 0.0 \% & 1 & 1.1 \%\end{array}$

$\begin{array}{llllll}143 & 54.6 \% & 34 & 35.8 \% & 57 & 63.3 \%\end{array}$

$\begin{array}{llllll}117 & 44.7 \% & 61 & 64.2 \% & 31 & 34.4 \%\end{array}$

$\begin{array}{llllll}2 & 0.8 \% & 0 & 0.0 \% & 2 & 2.2 \%\end{array}$

$\begin{array}{llllll}213 & 81.3 \% & 73 & 76.8 \% & 67 & 74.4 \%\end{array}$

$\begin{array}{llllll}46 & 17.6 \% & 22 & 23.2 \% & 15 & 16.7 \%\end{array}$

$\begin{array}{llllll}3 & 1.1 \% & 0 & 0.0 \% & 8 & 8.9 \%\end{array}$

$1.35 \quad 0.51$

$16.60<0.001^{* * *}$

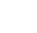

$$
3.18 \quad 0.20
$$

$\begin{array}{llllll}17 & 6.7 \% & 3 & 3.3 \% & 8 & 10.0 \%\end{array}$

$\begin{array}{llllll}237 & 93.3 \% & 89 & 96.7 \% & 72 & 90.0 \%\end{array}$

$\begin{array}{llllll}70 & 29.7 \% & 19 & 21.6 \% & 41 & 56.9 \%\end{array}$

$\begin{array}{llllll}166 & 70.3 \% & 69 & 78.4 \% & 31 & 43.1 \%\end{array}$ 
Table 2 Socio-demographic characteristic and sexual behavioural factors that associated with HIV testing among Chinese MSM (Continued)

HIV status of last sexual partners

Unaware

Aware

$$
\begin{aligned}
& \text { HIV-positive } \\
& \text { HIV-negative }
\end{aligned}
$$

\section{Homosexual behaviours (commercial)}

Had commercial sex partners in the past 6 months

$$
\text { No }
$$$$
\text { Yes }
$$

Used condom during the last anal intercourse

$$
\text { No }
$$$$
\text { Yes }
$$

HIV status of last sexual partners

$$
\begin{aligned}
& \text { Unaware } \\
& \text { Aware }
\end{aligned}
$$

\section{Heterosexual behaviours}

Had female sex partners in the past 6 months

$$
\begin{aligned}
& \text { No } \\
& \text { Yes }
\end{aligned}
$$

Used condom during the last vaginal intercourse

$$
\text { No }
$$$$
\text { Yes }
$$

HIV status of last sexual partners

$$
\begin{aligned}
& \text { Unaware } \\
& \text { Aware } \\
& \text { HIV-positive } \\
& \text { HIV-negative }
\end{aligned}
$$

\section{Multiple partnerships in the past 6 months}

Had both non-commercial male and female sex partners

$$
\begin{aligned}
& \text { No } \\
& \text { Yes }
\end{aligned}
$$

Used condom with non-commercial male and with female sex partners during the last anal/vaginal intercourse

$$
\text { No }
$$$$
\text { Yes }
$$

Had both non-commercial male and commercial male sex partners

$$
\begin{aligned}
& \text { No } \\
& \text { Yes }
\end{aligned}
$$

Used condom with non-commercial and commercial male sex partners during the last anal intercourse

$$
\text { No }
$$$$
\text { Yes }
$$

$\begin{array}{cccccc}127 & 53.8 \% & 49 & 55.7 \% & 49 & 69.0 \% \\ 109 & 46.1 \% & 39 & 44.3 \% & 22 & 31.0 \% \\ 2 & 0.8 \% & 2 & 2.3 \% & 0 & 0.0 \% \\ 107 & 45.3 \% & 37 & 42.0 \% & 22 & 31.0 \%\end{array}$

$3.96 \quad 0.14$

$\begin{array}{llllll}127 & 76.0 \% & 41 & 80.4 \% & 47 & 88.7 \%\end{array}$

$\begin{array}{llllll}40 & 24.0 \% & 10 & 19.6 \% & 6 & 11.3 \%\end{array}$

$0.47 \quad 0.79$

$\begin{array}{llllll}4 & 10.0 \% & 1 & 10.0 \% & 1 & 20.0 \%\end{array}$

$\begin{array}{llllll}36 & 90.0 \% & 9 & 90.0 \% & 4 & 80.0 \%\end{array}$

$\begin{array}{llllll}40 & 100 \% & 5 & 100 \% & 5 & 100.0 \%\end{array}$ $\begin{array}{llllll}0 & 0 \% & 0 & 0 \% & 0 & 0 \%\end{array}$

N/A N/A 
Table 2 Socio-demographic characteristic and sexual behavioural factors that associated with HIV testing among Chinese MSM (Continued)

Had both female and commercial male sex partners

No

Used condom with commercial male and with female sex partners during the last anal/vaginal intercourse

No

Yes

Had both non-commercial male, commercial male and female sex partners

No

Used condom with non-commercial male, commercial male and female sex partners during the last sexual intercourse

\begin{tabular}{|c|c|c|c|c|c|c|}
\hline No & 4 & $26.7 \%$ & 0 & $0 \%$ & 0 & $0 \%$ \\
\hline Yes & 11 & $73.3 \%$ & 2 & $100 \%$ & 0 & $0 \%$ \\
\hline
\end{tabular}

Note: ${ }^{*} p<0.05,{ }^{* *} p<0.01$ and ${ }^{* * *} p<0.001$.

$\wedge$ Chi-square test was performed between MSM who were aware their partners' HIV status (positive or negative) and those who were not.

tested within the past 12 months $(27.88 \pm 7.76)$ and more than 12 months ago $(30.17 \pm 7.85)(F=4.80, p=$ 0.03). In terms of marital status, the majority of nevertested MSM (80.0\%) had never married compared with those who had tested within the past 12 months $(62.2 \%)$ and more than 12 months ago $(54.7 \%)\left(\chi^{2}=16.88, p<\right.$ 0.001). Furthermore, a significantly higher proportion of students had never tested for HIV compared with those who were employed $\left(x^{2}=16.60, p<0.001\right)$. There was no significant association between self-identified sexual identify and testing behaviours $\left(\chi^{2}=1.35, p=0.51\right)$. No significant association between HIV testing behaviours and residency of the study participants $\left(x^{2}=3.39, p=\right.$ 0.18 ) was observed.

\section{Trend, frequency and barriers of HIV testing}

Figure 1 showed that the annual HIV testing rate among MSM significantly increased from $16.6 \%(74 / 447)$ in 2009 to $46.3 \%(207 / 447)$ in 2010 to $58.6 \%(262 / 447)$ in $2011\left(\chi^{2}=173.49, p<0.001\right)$. Among those who have been tested, a substantial proportion had been tested more than once in 2009 (29.7\%, 22/74); 2010 (48.3\%, 100/207); and 2011 (45.4\%, 119/262). In total, 879 HIV tests had been conducted among 357 ever-tested MSM

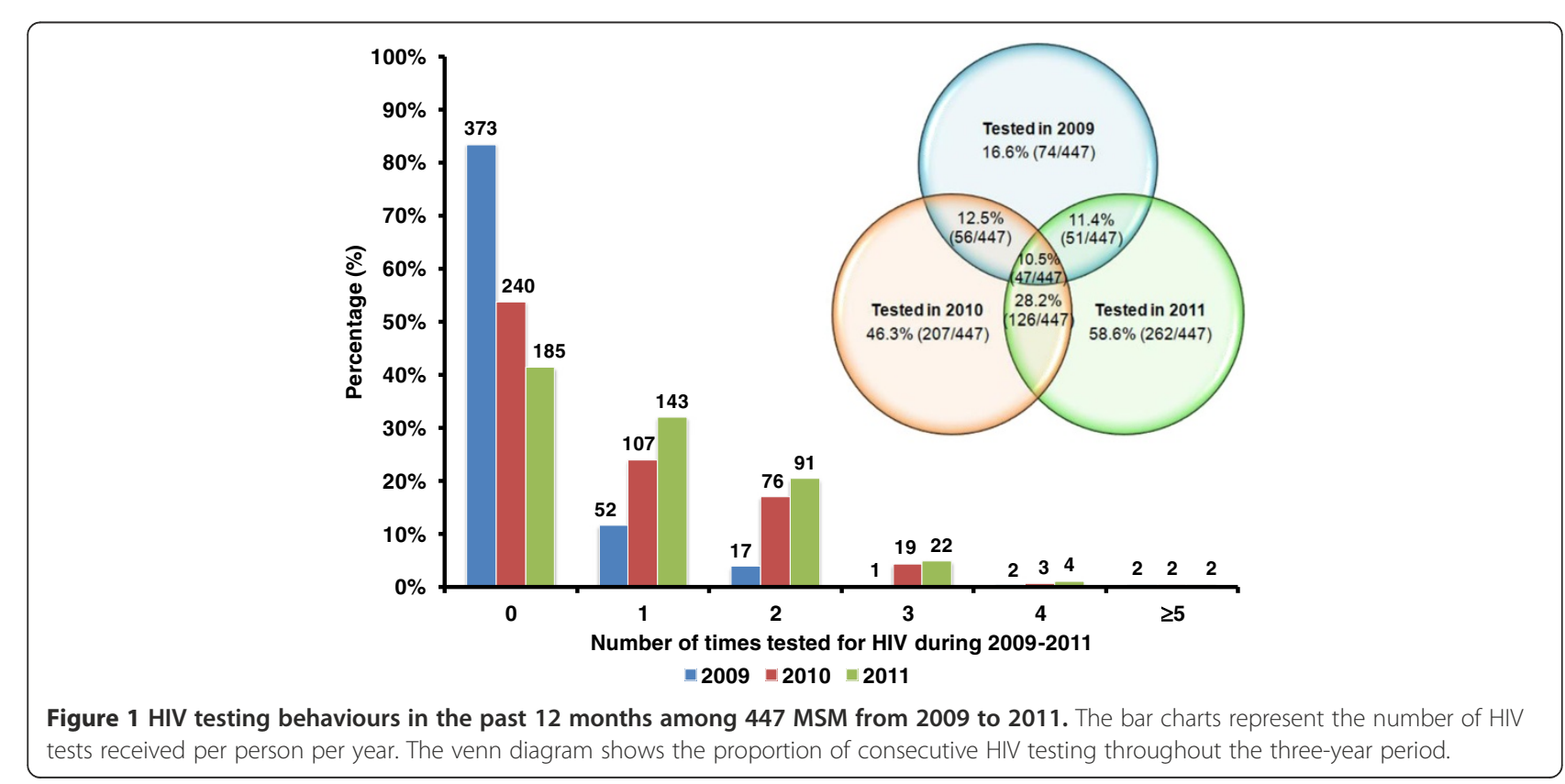


during 2009-2011. Of this group, only 47 MSM had received at least one HIV test annually throughout the three consecutive years. Additionally, there was a slight decline in HIV testing incidence from 2010 (38.4 cases per 100 population) to 2011 (34.1 cases per 100 population), but the change was not significant $\left(x^{2}=1.46, p=\right.$ 0.228 ). Approximately $8.1 \%$ (29/357) participants selfreported of not knowing their current HIV serostatus. Of these 29 MSM, 16 had tested more than once during this period.

Psychological and structural barriers to HIV testing among the ninety never-tested MSM are outlined in Table 3. The major psychological barriers were selfperception as healthy (55.6\%); perceived low risk of HIV infection due to consistent condom use (30.0\%); and the fear of discovering their own serostatus (27.8\%). Other reasons such as only having had sex with healthy-looking partners (18.9\%); fear of exposing their sexual orientation (17.8\%); having sex with regular partners only (14.4\%); and potential social stigma due to diagnosis of HIV/AIDS (11.1\%) were also reported as psychological barriers. Structural barriers to HIV testing included not knowing where to get tested (33.3\%), real-name registration testing (31.1\%) and concerns about confidentiality (31.1\%).

\section{Multiple sexual partnerships}

Most MSM (93.4\%, 398/426) had non-commercial maleto-male sex in the past six months. Our results indicated that the majority of never-tested MSM did not use condoms in the last sex act (56.9\%), doubled the percentage of those who had tested in the past 12 months (29.7\%) and those who tested more than 12 months ago (21.6\%); the difference between three groups were highly significant $\left(\chi^{2}=25.10, p<0.001\right)$. Furthermore, less than half of ever-tested MSM $(45.7 \%, 148 / 324)$ and never-tested MSM (31.0\%) were aware of the HIV serostatus of their last non-commercial male partners. Among the 349 participants who reported non-commercial male-to-male sex, the average duration between the last two consecutive episodes of sexual intercourse with non-commercial partners was estimated to be 6.53 (95\% CI 5.92-7.14) days (Figure 2).

About one-fifth of MSM (20.7\%; 56/271) had male-tomale commercial (including both patronizing and selling) sex in the past six months. Higher levels of condom use with commercial sex partners were reported among both MSM who had tested in the past 12 months $(90.0 \%, 36 /$ 40) and those who had tested more than 12 months ago (90.0\%, 9/10), compared to those never-tested MSM $(80.0 \%, 4 / 5)$; but the difference was not statistically significant $\left(x^{2}=0.47, p=0.79\right)$. Among the 56 participants who reported commercial male-to-male sex, all of them were ignorant of the HIV serostatus of their last commercial male sex partner. The duration between the last two consecutive episodes of commercial sex was approximately 1.17 (95\% CI 0.61-1.74) days (Figure 2). Notably, more than one-third of the MSM (36.0\%) had two or more episodes of commercial sex on the day of survey.

Our results showed that about $24.8 \%(67 / 270)$ of MSM had female sex partners in the past six months. Lower rate of condom use with female partners was reported among never-tested MSM $(18.2 \%, 2 / 11)$ compared to those who

Table 3 Reasons for not having HIV testing among MSM

\begin{tabular}{|c|c|c|}
\hline Reasons for not having HIV testing & $\mathbf{N}$ & \%* \\
\hline \multicolumn{3}{|l|}{ Psychological barriers } \\
\hline Perceive myself are healthy & 50 & $55.6 \%$ \\
\hline Always have consistent condom use during any sexual acts & 27 & $30.0 \%$ \\
\hline Fear of discovering own HIV status & 25 & $27.8 \%$ \\
\hline Only have sex with people who look healthy & 17 & $18.9 \%$ \\
\hline Fear to expose my sexual orientation & 16 & $17.8 \%$ \\
\hline Have sex with regular partners only & 13 & $14.4 \%$ \\
\hline Diagnosis of HIV would lead to discrimination and psychological burden & 10 & $11.1 \%$ \\
\hline AIDS cannot be cured, cannot do anything if positive & 1 & $1.1 \%$ \\
\hline \multicolumn{3}{|l|}{ Structural barriers } \\
\hline Don't know where to go for HIV testing & 30 & $33.3 \%$ \\
\hline Need to provide real name for HIV testing & 28 & $31.1 \%$ \\
\hline Doubt about the confidentiality of Department of Health & 28 & $31.1 \%$ \\
\hline Time clash between working hours and HIV testing & 5 & $5.6 \%$ \\
\hline Attitudes of medical staff at testing sites are bad & 1 & $1.1 \%$ \\
\hline Other reasons & 11 & $12.2 \%$ \\
\hline
\end{tabular}

*Data and percentage were obtained and calculated from 90 MSM who never had an HIV test. 


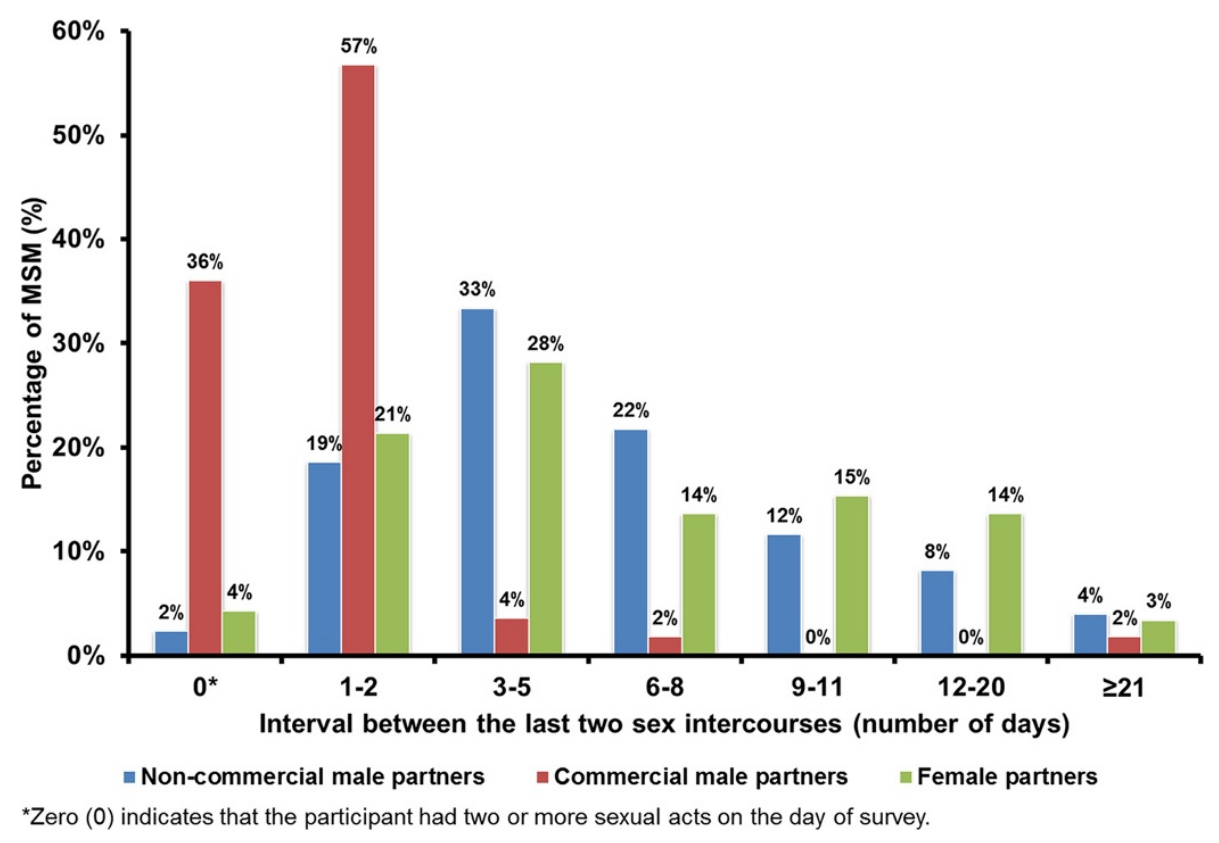

Figure 2 Distribution of sexual acts frequency between MSM with non-commercial male, commercial male and female partners.

had tested within the past 12 months $(50.0 \%, 20 / 40)$ and those who had tested more than 12 months ago $(35.7 \%, 5 / 14)$; however, the difference was not significant $\left(X^{2}=3.85, p=0.15\right)$. Significant association between HIV testing behaviours and the awareness of HIV serostatus of the last female sexual partner was observed $\left(x^{2}=9.58\right.$, $p=0.05)$. Among the 62 participants who reported noncommercial sexual activity with a female partner, the interval between the last two consecutive episodes of heterosexual sex was 7.53 (95\% CI 6.06-9.00) days (Figure 2).

Figure 3 illustrates the multiple type of sexual partnership among MSM with different types of self-identified sexual orientation. Self-identified heterosexual $(92.3 \% ; 12 / 13)$ and bisexual $(20.0 \% ; 10 / 50)$ MSM were more likely to have commercial male partners in the past six months than selfidentified homosexual men (16.1\%; 33/205). Overall, about $21.3 \%(56 / 263)$ of MSM reported having both noncommercial male and female sexual partnerships and about $6.2 \%(16 / 257)$ of MSM reported having all three types of sexual partnerships in the past six months.

\section{Discussion}

Our study demonstrates a significant increase in the HIV testing rate in the past 12 months among Chinese MSM from $16.6 \%$ to $58.6 \%$ during $2009-2011$. The increase may be due to the ongoing expansion of the national 'Four Frees and One Care' program which provides free voluntary HIV screening tests [23]. Despite the increase in testing coverage, the proportion of MSM who test regularly remains extremely low, as only $13.2 \%(47 / 357)$ of evertested MSM have tested repeatedly for HIV in three consecutive years. Consistent with previous findings $[24,25]$, MSM who are not aware their sexual partners' HIV serostatus and those who have unprotected sexual intercourse at the last male-to-male sex act are more likely to take up HIV testing. In contrast, MSM who were younger, including those who are students or never married, are less likely to have been tested for HIV infection. Consistently, previous studies indicated that marital status may be a confounding factor of age [26,27], and the HIV higher testing rate among married MSM may be due to their more conservative perception of risk of HIV infection [28]. Among young students, insufficient HIV/AIDS knowledge and awareness due to inadequate sex education at school may contribute the low testing uptake [29]. As younger MSM are more sexually active and risk-taking in their sexual behaviours $[30,31]$, intervention programs that target young MSM to improve HIV awareness and serostatus disclosure before and after entering marriage are essential.

Self-perception as 'healthy' or at 'low risk of HIV infection' are the main reasons for not having regular HIV testing in our study participants. This is consistent with findings in other large urban cities such as Beijing $[24,28]$ and Shanghai [32]. The low-risk perception may explain the reported risk behaviours such as low condom use with male (43.1\%) and female (18.2\%) partners during the last sexual episode. Together with their low awareness of their sexual partners' HIV serostatus, these behavioural patterns would substantially facilitate the effective transmission of HIV infection. Increasing perception of HIV risk and safe sexual practices remain a major task in HIV prevention among Chinese MSM [24]. 


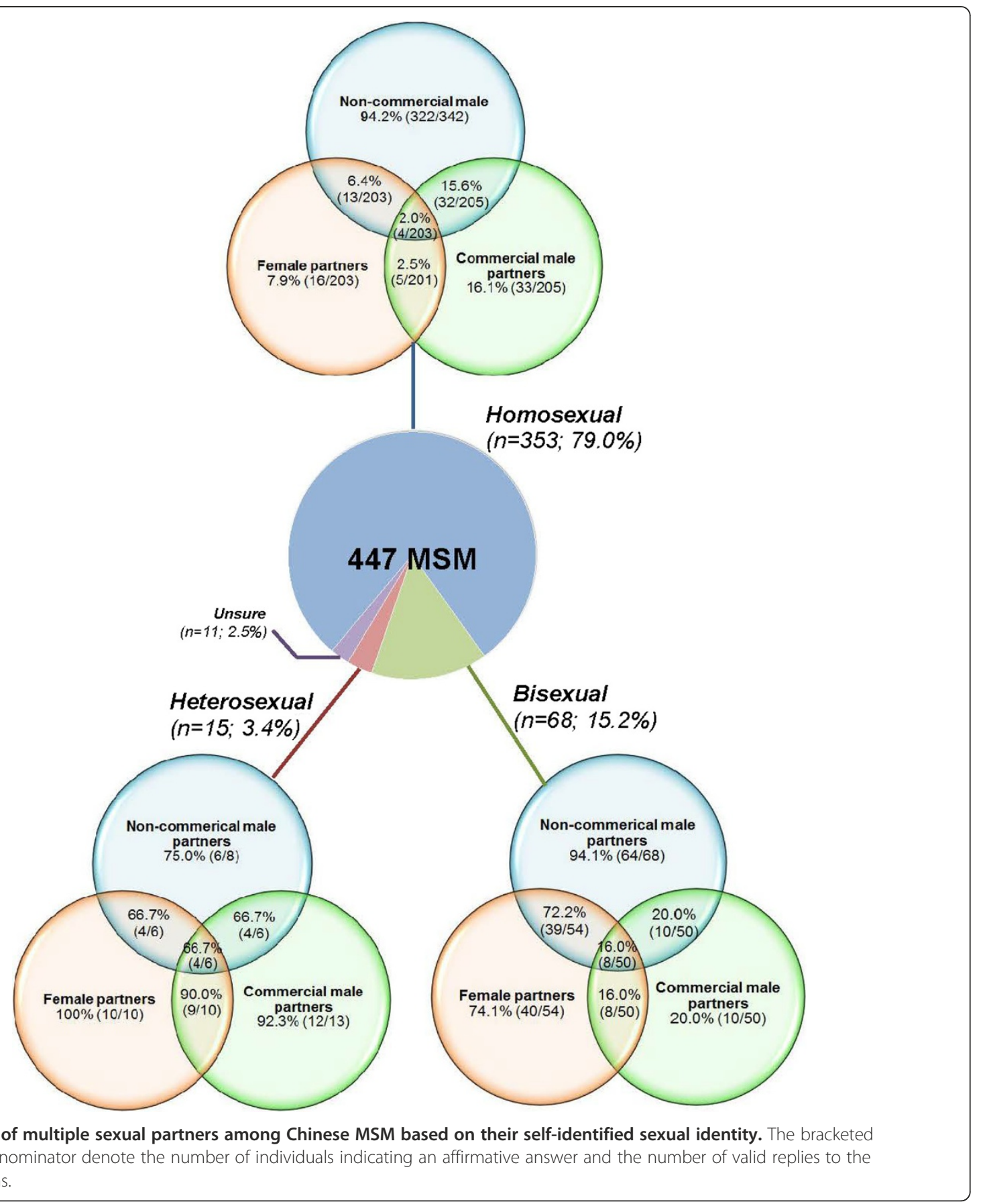

Bisexual behaviours are common among our study participants as approximately one-fifth of the MSM (21.3\%) reported having both male and female sexual partners in the past six months. This figure is slightly higher than previous studies conducted in Shanghai city $(15-17 \%)$ [33,34] but is lower than in Shenzhen city $(\sim 38 \%)$ in Southern China [35]. Our result showed that older MSM are more likely to have entered heterosexual marriage and to have unprotected sex with their female partners [6,36,37], indicating the importance of HIV testing in preventing the spread of HIV through both homosexual and heterosexual partnerships. Among bisexual MSM, about 29.1\% also participated in commercial sex with a male partner in the past six months. Bisexual MSM have sex with females as often as every 7.53 days, which is only slightly longer than the gap of homosexual intercourses with regular male partners (6.53 days). A meta-analysis showed that Chinese MSM with both male/female sex partners had significantly higher HIV prevalence than those with only male partners [38]. This strongly suggests that bisexual behaviours among 
MSM represent a very significant channel of HIV transmission to the female population [14].

Several limitations in this study should be noted. First, self-reporting and recall bias may have occurred in this retrospective study that requires recall of sexual behaviours in the past six months and the number of HIV tests received over the past three years. Participants might be inclined to provide socially desirable responses to the interviewers. Self-reporting bias may have occurred due to the varied demographic, ethnicity, level of education and culture. However, such association was not investigated in this study and hence caution is needed in interpreting results. In addition, the attitude and pattern of HIV testing could also be biased in different recruitment venues [39]. Second, as this study was conducted through both convenience and snowball sampling methods in two Chinese major cities, the mixed sampling method may affect the representativeness and ability to generalise from the sample to the broader MSM population in China.

Our study has several important implications for public health interventions and policies in China. First, scaling-up HIV testing efforts among younger MSM is a priority for effective HIV prevention among Chinese MSM. As this sexually active subgroup enters heterosexual marriage, bisexual behaviour of these individuals is likely to pose a significant risk in transmitting HIV infection to the low-risk female population $[36,40]$. Second, health promotion that aims to reduce psychological and structural barriers to HIV testing should be expanded among Chinese MSM. In addition to the traditional approaches of distributing educational materials through peer educators and at MSM hotspots, the internet has become an effective channel in disseminating relevant health information [22]. HIV pre- and post-test counselling provides unique opportunities to reduce the misconceptions about HIV infection and stigmatisation against people living with HIV (PLHIV) [41]. Third, recent meta-analyses indicated that behavioural interventions could effectively increase the uptake of HIV testing among Chinese MSM, but the majority of these interventions were only implemented in HIV-prevalent Southwest China [42,43]. Similar intervention programs could be expanded to other Chinese regions. Fourth, a substantial proportion (8.1\%) of ever-tested study participants had not been notified of their test results. Strategic positioning of new HIV testing sites may substantially increase accessibility, and the roll-out of rapid HIV testing would assist in MSM receiving immediate screening results [28]. Furthermore, the use of innovative and proved approaches (i.e. automated SMS [short message service], computer pop-up reminders and alerts) may also increase the return for repeated HIV testing and timely notifications of test results $[44,45]$.

\section{Conclusions}

HIV testing rate has increased among Chinese MSM but remains relatively low in comparison to those in developed countries settings. In addition, multiple sexual partnerships with both male and female partners among Chinese MSM may facilitate HIV transmission in MSM community and into the general female population. Specific recommendations have been made, including scaling up HIV testing campaigns and health promotion interventions, in order to increase the coverage and reduce barriers of HIV testing among Chinese MSM.

\section{Additional files}

Additional file 1: Questionnaire. HIV testing behaviour among MSM in China.

Additional file 2: Table S1. Socio-demographic characteristic factors that associated with HIV testing among Chinese MSM, stratified by cities (a) Changsha, and (b) Tianjin.

\section{Competing interests}

All authors declare that they have no competing interest.

\section{Authors' contributions}

EPFC participated in the study design, undertook the statistical analyses, wrote the first draft of the manuscript and was the primary author of this manuscript. JJ participated in the study design and revised the manuscript. YF obtained funding, critically reviewed and revised the manuscript. DM obtained funding and involved in the study design. JZ involved in the study design. DPW assisted in the study design and interpretation of results, critically reviewed and revised the manuscript. XZ participated in the data collection. LZ led the study, provided oversight in the study design and data analyses, critically reviewed and approved the final version of the manuscript. All authors contributed to and have approved the final version of the manuscript.

\section{Acknowledgment}

We thank all the participants in this study for their time and generously helping us in this research. We thank the following organizations for their grants: the Australian Government Department of Health and Ageing, the University of New South Wales, the Endeavour Research Fellowship (Award ID: 2744_2012) for its support in EPFC's study in China, Australian Postgraduate Awards, the World Bank Global HIV/AIDS Program, the Australian Research Council (Grant No.: FT0991990), and the Bill \& Melinda Gates Foundation. We would also like to thank Ms. Louisa Wright for her support in proofreading.

\section{Author details}

${ }^{1}$ The Kirby Institute, University of New South Wales, Sydney, New South Wales, Australia. ${ }^{2}$ Comprehensive AIDS Research Center, Tsinghua University, Beijing, China. ${ }^{3}$ Melbourne Sexual Health Centre, Alfred Hospital, Melbourne, Victoria, Australia. ${ }^{4}$ Central Clinical School, Faculty of Medicine, Nursing and Health Sciences, Monash University, Melbourne, Victoria, Australia. ${ }^{5}$ Bill \& Melinda Gates Foundation, Beijing Representative Office, Beijing, China. ${ }^{6}$ China Food and Drug Administration Institute of Executive Development, Beijing, China.

Received: 21 April 2013 Accepted: 7 November 2013 Published: 16 November 2013

\section{References}

1. Zhang L, Chow EP, Jing J, Zhuang X, Li X, He M, Sun H, Li X, Gorgens M, Wilson D, et al: HIV prevalence in China: integration of surveillance data and a systematic review. Lancet 2013, 13(11):955-963. 
2. State Council AIDS Working Committee Office (SCAWCO): China 2010 UNGASS Country Progress Report. Beijing, China: Ministry of Health of the People's Republic of China; 2010.

3. State Council AIDS Working Committee Office (SCAWCO): UNGASS Country Progress Report P. R. China (2006-2007). Beijing, China: Ministry of Health of the People's Republic of China; 2008.

4. Chow EP, Wilson DP, Zhang J, Jing J, Zhang L: Human immunodeficiency virus prevalence is increasing among men who have sex with men in China: findings from a review and meta-analysis. Sex Transm Dis 2011, 38(9):845-857.

5. State Council AIDS Working Committee Office (SCAWCO): 2012 China AIDS Response Progress Report. Beijing, China: Ministry of Health of the People's Republic of China; 2012:70.

6. Zhang $L$, Chow EPF, Wilson DP: Men who have sex with men in China have relatively low numbers of sexual partners. Infect Dis Rep 2011, 3(e10):46-51.

7. Chow EP, Wilson DP, Zhang L: What is the potential for bisexual men in China to act as a bridge of HIV transmission to the female population? Behavioural evidence from a systematic review and meta-analysis. BMC Infect Dis 2011, 11:242.

8. Chow EP, Iu Kl, Fu X, Wilson DP, Zhang L: HIV and sexually transmissible infections among money boys in China: a data synthesis and metaanalysis. PLoS One 2012, 7(11):e48025

9. Zhang B, Li X, Hu T, Liu D, Shi T: HIV/AIDS interventions targeting men who have sex with men (MSM): theory and practice. Chin J STD/AIDS Prev Cont 2000, 6(3):155-157.

10. Ruan Y, Li D, Li X, Qian HZ, Shi W, Zhang X, Yang Z, Wang C, Liu Y, Yu M, et al: Relationship between syphilis and HIV infections among men who have sex with men in Beijing, China. Sex Transm Dis 2007, 34(8):592-597.

11. Chow EP, Gao L, Koo FK, Chen L, Fu X, Jing J, Wilson DP, Zhang L: Qualitative exploration of HIV-related sexual behaviours and multiple partnerships among Chinese men who have sex with men living in a rural area of Yunnan Province, China. Sex Health 2013. [Epub ahead of print]. doi: 10.1071/SH13097.

12. Chow EP, Wilson DP, Zhang L: Patterns of condom use among men who have sex with men in China: a systematic review and meta-analysis. AIDS Behav 2012, 16(3):653-663.

13. Chow EP, Wilson DP, Zhang L: Estimating HIV incidence among female partners of bisexual men in China. Int J Infect Dis 2012, 16(5):e312-320.

14. Chow EP, Koo FK, Zhang L: Are wives of gay men becoming the next target of HIV infection in China? Sex Transm Dis 2013, 40(12):964-965.

15. Wilson DP, Hoare A, Regan DG, Law MG: Importance of promoting HIV testing for preventing secondary transmissions: modelling the Australian HIV epidemic among men who have sex with men. Sex Health 2009, 6(1):19-33.

16. National Centre in HIV Social Research: HIV/AIDS, Hepatitis and Sexually Transmissible Infections in Australia - Annual Report of Trends in Behaviour 2010. Sydney: National Centre in HIV Social Research, University of New South Wales; 2010

17. Norwegian Directorate of Health: UNGASS Country Progress Report Norway: Januan 2008-December 2009. Oslo, Norway: Norwegian Directorate of Health; 2010.

18. Chow EP, Wilson DP, Zhang L: The next era of HIV in China: rapidly spreading epidemics among men who have sex with men. J Acquir Immune Defic Syndr 2010, 55(4):e32-33. author reply e33-34.

19. MacKellar DA, Valleroy LA, Secura GM, Behel S, Bingham T, Celentano DD, Koblin BA, Lalota M, McFarland W, Shehan D, et al: Unrecognized HIV infection, risk behaviors, and perceptions of risk among young men who have sex with men: opportunities for advancing HIV prevention in the third decade of HIV/AIDS. J Acquir Immune Defic Syndr 2005, 38(5):603-614.

20. Buchbinder S: The epidemiology of new HIV infections and interventions to limit HIV transmission. Top HIV Med 2009, 17(2):37-43.

21. Williamson LM, Dodds JP, Mercey DE, Hart GJ, Johnson AM: Sexual risk behaviour and knowledge of HIV status among community samples of gay men in the UK. AIDS 2008, 22(9):1063-1070.

22. Holt M, Rawstorne P, Wilkinson J, Worth H, Bittman M, Kippax S: HIV testing, gay community involvement and internet use: social and behavioural correlates of HIV testing among Australian men who have sex with men. AIDS Behav 2012, 16(1):13-22.

23. State Council AIDS Working Committee Office, UN Theme Group on HIV/AIDS in the People's Republic of China: A Joint Assessment of HIV/AIDS Prevention, Treatment and Care in China. Beijing: China Ministry of Health; 2004.

24. Song Y, Li X, Zhang L, Fang X, Lin X, Liu Y, Stanton B: HIV-testing behavior among young migrant men who have sex with men (MSM) in Beijing, China. AIDS Care 2011, 23(2):179-186.
25. Wei C, Ruan S, Zhao J, Yang H, Zhu Y, Raymond HF: Which Chinese men who have sex with men miss out on HIV testing? Sex Transm Infect 2011, 87(3):225-228

26. Shi T, Zhang B, Li X, Xu J, Wang N, Zhou S, Guan W, Zhang J: Comparison of AIDS related high risk behaviors between married and unmarried men who have sex with men. Chin JAIDS STD 2008, 14(5):475-478.

27. Guo H, Wu ZY: Review of study on marital status and sex behavior features of men who have sex with men. Dis Surveill 2010, 25(8):651-654.

28. Choi KH, Lui H, Guo Y, Han L, Mandel JS: Lack of HIV testing and awareness of HIV infection among men who have sex with men, Beijing, China. AIDS Educ Prev 2006, 18(1):33-43.

29. Zhao EJ, Cui D, Liang SY, Guo JL, Wang YM, Lu WQ: Meta-analysis of effectivness of AIDS health education among college students. Mod Prev Med 2011, 38(20):4105-4109.

30. Feng LG, Ding XB, Lu RR, Xu SM, Guo XJ, Yang MF, Zheng JQ: Trend on prevalence of HIV and syphilis and estimated HIV incidence among young male students who have sex with men. Acta Academiae Medicinae Militaris Tertiae 2010, 32(24):2644-2646.

31. Zheng YF, Pan CE, Zhang RF, Liu L, Chen YZ, Wang JR, Jiang XY, Qi TK, Lu $\mathrm{HZ}$ : A clinical epidemiological study on AIDS patients attributed to maleto-male sexual contact in Shanghai. Chin J AIDS STD 2011, 17(5):523-525.

32. Huang ZJ, He N, Nehl EJ, Zheng T, Smith BD, Zhang J, McNabb S, Wong FY: Social network and other correlates of HIV testing: findings from male sex workers and other MSM in Shanghai, China. AIDS Behav 2012, 16(4):858-871.

33. Choi KH, Hudes ES, Steward WT: Social discrimination, concurrent sexual partnerships, and HIV risk among men who have sex with men in Shanghai, China. AIDS Behav 2008, 12(4 Suppl):S71-77.

34. Choi KH, Ning Z, Gregorich SE, Pan QC: The influence of social and sexual networks in the spread of HIV and syphilis among men who have sex with men in Shanghai, China. J Acquir Immune Defic Syndr 2007, 45(1):77-84.

35. Su QX, Zhou F, Tian XB, Zhang KL, Wu ZL: Capacity of non-governmental organizations of men who have sex with men at grassroots involving in HIV/ AIDS prevention and control. Chin J Dis Contr Prevent 2010, 14(4):296-299.

36. Ruan S, Yang H, Zhu Y, Wang M, Ma Y, Zhao J, McFarland W, Raymond HF: Rising HIV prevalence among married and unmarried among men who have sex with men: Jinan, China. AIDS Behav 2009, 13(4):671-676.

37. He Q, Wang Y, Lin P, Liu Y, Yang F, Fu X, Li Y, Sun B, Li J, Zhao X, et al: Potential bridges for HIV infection to men who have sex with men in Guangzhou, China. AIDS Behav 2006, 10(4 Suppl):S17-23.

38. Yun K, Xu JJ, Reilly KH, Zhang J, Jiang YJ, Wang N, Shang H: Prevalence of bisexual behaviour among bridge population of men who have sex with men in China: a meta-analysis of observational studies. Sex Transm Infect 2011, 87(7):563-570.

39. Department of Health and Human Services Centers for Disease Control and Prevention: Prevalence and Awareness of HIV Infection Among Men Who Have Sex With Men - 21 cities, United States, 2008. MMWR Morb Mortal Wkly Rep 2010, 59(37):1201-1207.

40. Chow EP, Wilson DP, Zhang L: The rate of HIV testing is increasing among men who have sex with men in China. HIV Med 2012, 13(5):255-263.

41. Wang $C H$, Pang $L$, Wu $Z Y$ : The impact of voluntary counselling and testing (VCT) on HIV/AIDS prevention. Chin J AIDS STD 2004, 10(6):471-473.

42. Zheng $L$, Zheng $Y$ : Efficacy of human immunodeficiency virus prevention interventions among men who have sex with men in China: a metaanalysis. Sex Transm Dis 2012, 39(11):886-893.

43. Huang Z, Wang M, Fu L, Fang Y, Hao J, Tao F, Tu C: Intervention to increase condom use and HIV testing among men who have sex with men in China: a meta-analysis. AIDS Res Hum Retroviruses 2013, 29(3):441-448.

44. Zou H, Fairley CK, Guy R, Chen MY: The efficacy of clinic-based interventions aimed at increasing screening for bacterial sexually transmitted infections among men who have sex with men: a systematic review. Sex Transm Dis 2012, 39(5):382-387.

45. Zou H, Wu Z, Yu J, Li M, Ablimit M, Li F, Poundstone K: Internet-facilitated voluntary counseling and testing (VCT) clinic-based HIV testing among men who have sex with men in China. PloS One 2013, 8(2):e51919.

doi:10.1186/1471-2334-13-549

Cite this article as: Chow et al:: Pattern of HIV testing and multiple sexual partnerships among men who have sex with men in China. BMC Infectious Diseases 2013 13:549. 